\title{
FrHPI: A Discriminative Patch-Image Model for Hyperspectral Anomaly Detection
}

\author{
Hao Li $\mathbb{D}^{1}{ }^{1}$ Ganghui Fan, ${ }^{2}$ Shan Zeng, ${ }^{1}$ and Zhen Kang ${ }^{1}$ \\ ${ }^{1}$ School of Mathematics and Computer Science, Wuhan Polytechnic University, Wuhan 430023, China \\ ${ }^{2}$ School of Electrical and Electronic Engineering, Wuhan University, Wuhan 430023, China \\ Correspondence should be addressed to Hao Li; lihao@whpu.edu.cn
}

Received 12 August 2020; Revised 22 September 2020; Accepted 10 March 2021; Published 23 March 2021

Academic Editor: Xiaojie Guo

Copyright (C) 2021 Hao Li et al. This is an open access article distributed under the Creative Commons Attribution License, which permits unrestricted use, distribution, and reproduction in any medium, provided the original work is properly cited.

\begin{abstract}
Anomaly detection is now a significantly important part of hyperspectral image analysis to detect targets in an unsupervised manner. Traditional hyperspectral anomaly detectors fail to consider spatial information, which is vital in hyperspectral anomaly detection. Moreover, they usually take the raw data without feature extraction as input, limiting the detection performance. We propose a new anomaly detector based on the fractional Fourier transform (FrFT) and a modified patch-image model called the hyperspectral patch-image (HPI) model to tackle these two problems. By combining them, the proposed anomaly detector is named fractional hyperspectral patch-image (FrHPI) detector. Under the assumption that the target patch-image is a sparse matrix while the background patch-image is a low-rank matrix, we first formulate a matrix by sliding a rectangle window on the first three principal components (PCs) of HSI. The matrix can be decomposed into three parts representing the background, targets, and noise with the well-known low-rank and sparse matrix decomposition (LRaSMD). Then, distinctive features are extracted via FrFT, a transformation which is desirable for noise removal. Background atoms are selected to construct the covariance matrix. Finally, anomalies are picked up with Mahalanobis distance. Extensive experiments are conducted to verify the proposed FrHPI detector's superiority in hyperspectral anomaly detection compared with other state-of-the-art detectors.
\end{abstract}

\section{Introduction}

Hyperspectral imagery (HSI), with hundreds of narrow bands, can provide more abundant spectral information than other remote sensing approaches, such as infrared images and multispectral images [1]. Exploiting this property, HSI shows its advantage in classification, unmixing, and target detection [2-4], which has been widely employed in many fields, including intelligent agriculture, mineral exploration, and military applications [5-10]. As an essential part in hyperspectral image analysis, anomaly detection aims at identifying targets in an unsupervised manner, which possesses the following characteristics: (1) the spectral curves of anomalies are different from those of the surrounding background, and they only occupy a tiny part of the entire image; (2) no spectral information about background or targets are known in advance. Consequently, hyperspectral anomaly detection is now a hot but challenging topic in the remote sensing domain [11-13]. Over the last few decades, various methods are proposed for hyperspectral anomaly detection, which can be divided into two categories: distance-based and representation-based.

Distance-based algorithms use the covariance matrix to model the background, assuming that the background obeys the multivariate Gaussian distribution. As a benchmark in hyperspectral anomaly detection, the Reed-Xiaoli (RX) [14] detector finds anomalies with Mahalanobis distance based on the generalized-likelihood ratio test (GLRT) and has derived a series of algorithms. Local RX [15] adopts a dualwindow strategy to exploit the local spatial information. The kernel version [16] was proposed based on the kernel theory by considering the high-order relationships among spectral bands. Distance-based anomaly detectors are comprehensible and straightforward, which have been employed in many remote sensing tasks. However, the Gaussian 
distribution assumption can be violated in some complicated scenes, thus degrading detection performance [17].

Representation-based algorithms utilize the property that anomalies usually behave differently from the background. These representation-based algorithms do not need to assume the probability distribution and show its advantage in separating the background from noise and anomalies. Collaborative-representation-based detector (CRD) [18] assumes that the background can be represented by surrounding pixels while anomalies cannot, and it is now a popular technology in hyperspectral image processing. Low-rank and sparse matrix decomposition (LRaSMD) takes full advantage of the low-rank property in the background and the sparsity maintains in targets, which gets much attention recently $[19,20]$. Low-rank and sparse representation (LRASR) [21] detector decomposes HSI into three parts representing the background, targets, and noise. In [22], Zhang et al. integrated the low-rank prior knowledge of the background into LRaSMD to set background apart from anomalies. $\mathrm{Xu}$ [23] also used the sparse matrix, and the final detection result is obtained by adding the low-rank part and spare part together with a ratio. Deep learning also shows its advantage in extracting intrinsic features from data, but it is time-consuming and needs labels to induct the network to adjust the weights [24, 25].

Due to the corruption of noise and low spatial resolution, the spectral curves may share similar patterns between background and anomalies, whereas the algorithms mentioned above reconstruct the background merely with the raw data, which limits the final detection performance. Moreover, the representation-based algorithms usually perform in pixel-level without consideration of spatial information. To address these problems, we propose a fractional Fourier transform (FrFT) and hyperspectral patchimage (HPI) model-based anomaly detector named fractional hyperspectral patch-image (FrHPI) detector. Inspired by the infrared image-patch (IPI) model for small target detection in infrared images [26], which utilizes the similarity among background patches and the sparsity of targets, we modify it as HPI for hyperspectral anomaly detection purpose. In terms of feature extraction, [27] introduced FrFT into hyperspectral anomaly detection, causing the anomalies to separate better from the background to improve the performance. Due to its good performance, we introduce FrFT to convert the data from the spectral domain into an intermediate domain between the original spectral domain and its Fourier transform domain for final detection.

The main contributions of this paper are listed as follows:

(1) The proposed HPI takes full advantage of the nonlocal self-correlation property of the background and the sparsity of anomalies, and the background is separated by solving the optimization problem of recovering low-rank and sparse matrices with the well-known LRaSMD, which serves as an indicator for background covariance matrix formulation.

(2) FrFT is employed as preprocessing in FrHPI for final anomaly detection, which is beneficial to highlighting the discrimination between background and anomalies. The detection map is obtained via Mahalanobis distance.

The remaining part of this paper proceeds as follows. In Section 2, the whole procedure and methods used for the proposed anomaly detector are introduced. Experiments with four HSI data sets are conducted in Section 3 to verify the efficiency and effectiveness of the FrHPI detector. Conclusions are drawn in Section 4.

\section{Procedure and Methods}

2.1. Procedure of FrHPI. As illustrated in Figure 1, the first three principal components (PCs) are obtained via the principal component analysis (PCA). Then, the proposed HPI model is used to separate the background from anomalies, and the background atoms with high confidence are selected to induce the formulation of the covariance matrix. Meanwhile, FrFT transforms the raw data into an intermediate domain between the signal domain and its Fourier domain to extract more distinctive features. Mahalanobis distance is used lastly to detect potential targets.

2.2. $L R a S M D$. The background is usually redundant in hyperspectral images and lies in a low-dimensional subspace, which possesses the low-rank property. On the contrary, anomalies are sparse because they occupy a relatively small part of the whole image. In light of this, LRaSMD decomposes the reshaped HSI into a low-rank matrix L representing background, a sparse matrix $S$ corresponding to anomalies, and a noise matrix V. Hyperspectral anomaly detection via LRaSMD is complicated by solving the optimization problem:

$$
\begin{aligned}
& \min _{\mathbf{L}, \mathbf{S}}, \operatorname{rank}(\mathbf{L})+\lambda \cdot \operatorname{card}(\mathbf{S}) \\
& \text { s.t, } \mathbf{X}=\mathbf{L}+\mathbf{S}+\mathbf{V},
\end{aligned}
$$

where $\operatorname{rank}(\mathbf{L})$ and $\operatorname{card}(\mathbf{S})$ are the rank and cardinality of matrix $\mathbf{L}$ and $\mathbf{S}$, respectively, and $\lambda$ is a positive tradeoff parameter to balance the influence of the two parts in (1). However, the rank function is nonconvex due to the discrete property. Fortunately, the nuclear norm (i.e., the sum of singular values) and $l_{1}$-norm are good surrogates. Thus, problem (1) can be replaced as

$$
\begin{gathered}
\min _{\mathbf{L}, \mathbf{S}}\|\mathbf{L}\|_{*}+\lambda\|\mathbf{S}\|_{1}, \\
\text { s.t, } \mathbf{X}=\mathbf{L}+\mathbf{S}+\mathbf{V} .
\end{gathered}
$$

The augmented Lagrange function of problem (2) is shown as follows:

$$
\begin{aligned}
& f(\mathbf{L}, \mathbf{S}, \mathbf{Y}) \\
& =\|\mathbf{L}\|_{*}+\lambda\|\mathbf{S}\|_{1}+<\mathbf{Y}, \mathbf{X}-\mathbf{L}-\mathbf{S}>+\frac{\mu}{2}\|\mathbf{X}-\mathbf{L}-\mathbf{S}\|_{F}^{2},
\end{aligned}
$$

wherein $\mathbf{Y}$ is a set of the Lagrange multipliers and $\mu$ is the penalty factor. The optimal solution for hyperspectral anomaly detection can be obtained by minimizing problem 


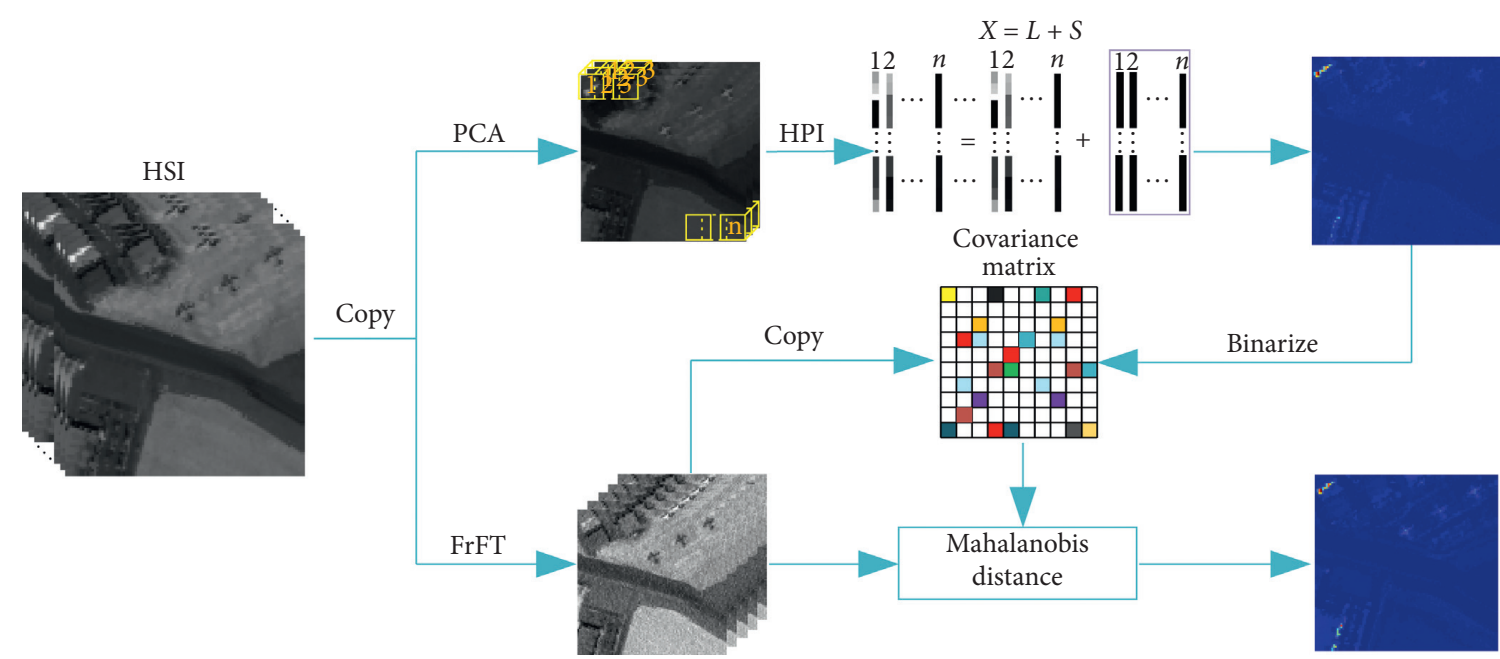

FIgURE 1: Flowchart of the proposed FrHPI detector.

(3), and the alternating direction method of multipliers (ADMMs) is widely used to solve this optimization problem.

(1) Fix $\mathbf{S}$ and $\mathbf{Y}$, and update $\mathbf{L}$. The objective function for $\mathbf{L}$ is written as follows:

$$
\begin{aligned}
\mathbf{L}^{k+1} & =\arg \min _{\mathbf{L}}\|\mathbf{L}\|_{*}+<\mathbf{Y}, \mathbf{X}-\mathbf{L}-\mathbf{S}^{k}>+\frac{\mu}{2}\left\|\mathbf{X}-\mathbf{L}-\mathbf{S}^{k}\right\|_{F}^{2} \\
& =\arg \min _{\mathbf{L}}\|\mathbf{L}\|_{*}+\frac{1}{2 / \mu}\left\|\mathbf{L}-\left(\mathbf{X}-\mathbf{S}^{k}+\frac{\mathbf{Y}^{k}}{\mu}\right)\right\|_{F}^{2} .
\end{aligned}
$$

Problem (4) can be solved via the singular value thresholding (SVT) [28]:

$$
\mathbf{L}^{k+1}=\mathbf{U}_{\mathbf{L}} T_{1 / \mu}\left(\Sigma_{\mathbf{L}}\right) \mathbf{V}_{\mathbf{L}}^{T}
$$

where

$$
\begin{aligned}
\left(\mathbf{X}-\mathbf{S}^{k}+\frac{\mathbf{Y}^{k}}{\mu}\right) & =\mathbf{U}_{\mathbf{L}} \Sigma_{\mathbf{L}} \mathbf{V}_{\mathbf{L}}^{T}, \\
T_{1 / \mu}\left(\Sigma_{\mathbf{L}}\right)_{i j} & = \begin{cases}\Sigma_{\mathbf{L} i j}-\frac{1}{\mu}, & \Sigma_{\mathbf{L} i j}>\frac{1}{\mu} \\
\Sigma_{\mathbf{L} i j}+\frac{1}{\mu}, & \Sigma_{\mathbf{L} i j}>\frac{1}{\mu} \\
0, & \text { otherwise. }\end{cases}
\end{aligned}
$$

(2) Fix $\mathbf{L}$ and $\mathbf{Y}$, and update $\mathbf{S}$. The objective function with respect to $\mathbf{S}$ is written as follows:

$$
\begin{aligned}
\mathbf{S}^{k+1} & =\arg \min _{\mathbf{S}}\|\mathbf{S}\|_{1}+<\mathbf{Y}^{k}, \mathbf{X}-\mathbf{L}^{k+1}-\mathbf{S}>+\frac{\mu}{2}\left\|\mathbf{X}-\mathbf{L}^{k+1}-\mathbf{S}\right\|_{F}^{2} \\
& =\arg \min _{\mathbf{S}}\|\mathbf{S}\|_{1}+\frac{1}{2 \lambda / \mu}\left\|\mathbf{S}-\left(\mathbf{X}-\mathbf{L}^{k+1}+\frac{\mathbf{Y}^{k}}{\mu}\right)\right\|_{F}^{2}
\end{aligned}
$$

Problem (8) can be solved via soft thresholding [29]:

$$
\mathbf{S}^{k+1}=T_{\lambda / \mu}\left(\mathbf{X}-\mathbf{L}^{k+1}+\frac{\mathbf{Y}^{k}}{\mu}\right)
$$

(3) Fix $\mathbf{L}$ and $\mathbf{S}$, and update $\mathbf{Y}$ with the gradient ascend method:

$$
\mathbf{Y}^{k+1}=\mathbf{Y}^{k}+\mu\left(\mathbf{X}-\mathbf{L}^{k+1}-\mathbf{S}^{k+1}\right)
$$

The optimal solution is obtained by alternately updating $\mathbf{L}, \mathbf{S}$, and $\mathbf{Y}$ until convergence.

2.3. FrFT. The Fourier transform (FT) converts the original data into its Fourier domain and is widely used in signal processing [30]. However, images captured from satellite or airborne sensors are affected by atmospheric conditions, variations of the material surface, and other influences [27], causing the FT performs poorly in many remote sensing tasks. Nevertheless, FrFT can better handle nonstationary noise than FT [31, 32], encouraging better separation between anomalies and background [33]. FrFT can be viewed as a transition between the original signal and its Fourier transform with an angle determined by fractional transform order $p$. For each pixel $\mathbf{x}_{i} \in R^{b}$ with $b$ spectral bands, its FrFT can be represented as

$$
\tilde{x}_{i}(v)=\frac{1}{b} \sum_{u=1}^{b} x_{i}(u) K_{p}(u, v),
$$

with

$$
\begin{aligned}
K_{p}(u, v) & =A_{\alpha} \exp \left\{j \pi\left(u^{2} \cot \alpha-2 u v \csc \alpha+v^{2} \cot \alpha\right)\right\} \\
A_{\alpha} & =\frac{\exp \{(-j \pi \operatorname{sgn}(\sin \alpha) / 4)+(j \alpha / 2)\}}{|\sin \alpha|^{1 / 2}}
\end{aligned}
$$

where $u$ and $v$ are indices, the range of fractional transform order $p$ is $[0,1]$ and the rotation angle $\alpha=p \pi / 2$. Figure 2 


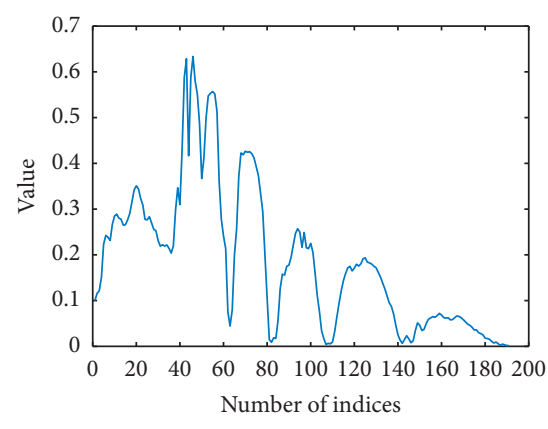

$-p=0$

(a)

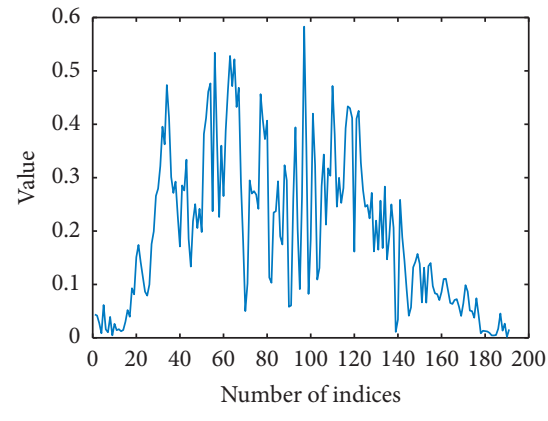

$-p=0.6$

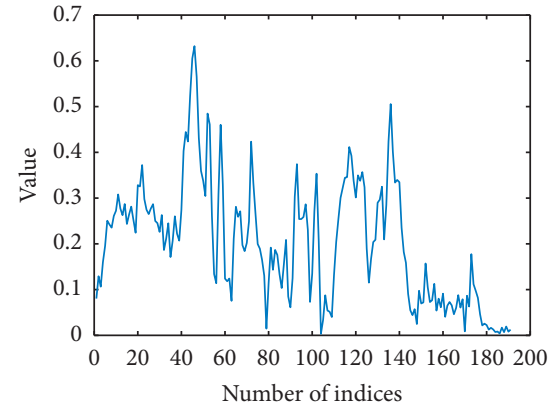

$-p=0.2$

(b)

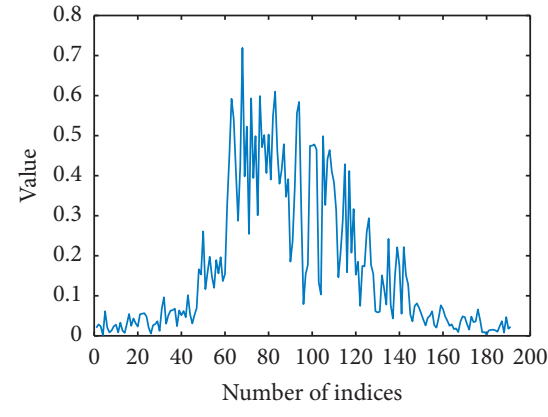

$\longrightarrow p=0.8$

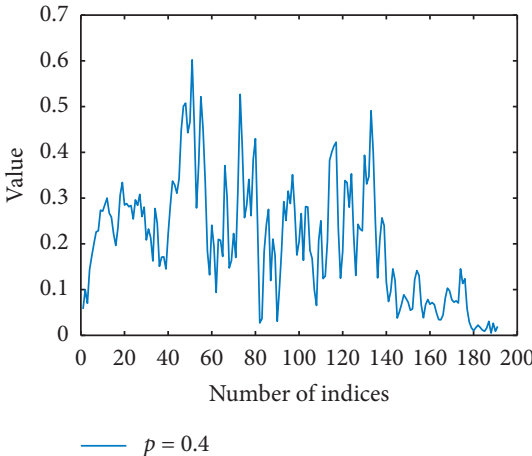

(c)

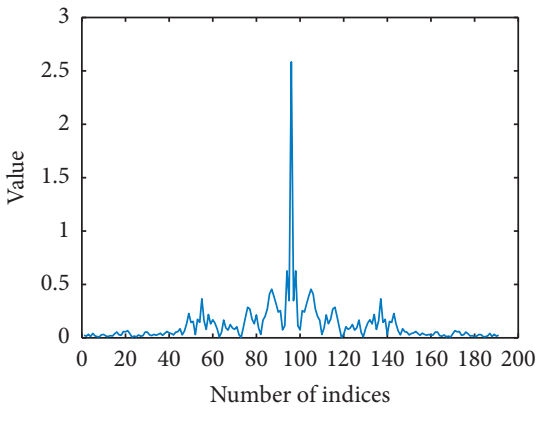

(f)

Figure 2: Illustration of how parameter $p$ influences the output of FrFT. (a-f) The outputs of FrFT when $p$ is equal to 0, 0.2, 0.4, 0.6, 0.8, and 1.0 , respectively.

briefly shows how fractional transform order $p$ influences the output of FrFT. With the increase of parameter $p$, the output tends to contain more Fourier domain information, and vice versa. Anomalies can be easier distinguished from a background in an intermediate domain with a proper setting of $p$. FrFT shows this property in hyperspectral detection tasks $[27,34]$, and the impact of it on the detection result will be analyzed in Section 3.3.

2.4. HPI. The Infrared patch-image (IPI) model is a kind of detector with high-performance in infrared target detection, which assumes that background possesses the nonlocal selfcorrelation property while anomalies are sparse among the whole images [27]. Experiments demonstrate its superiority in signal-to-clutter ratio (SCR) and background suppress factor (BSF) when compared with other traditional target detectors.

Inspired by the IPI model, we extract the first three PCs in advance, as these PCs own the most spatial information that can be utilized to improve the detection performance. Figure 3 demonstrates the procedure of the proposed HPI model. Initially, the first three PCs maintaining the most spatial information are extracted via PCA. Then, a series of local image patches are obtained with a sliding rectangle window with a size of $w \times w$ from the left top to the right down in these PCs, and the slide width is set to $s$. After this, we can get $n$ patches vectorized as columns of matrix $\mathbf{X}$, namely, the patch-images as defined in [22]. The size of patch-image $\mathbf{X}$ is sensitive to the sliding window size $w$. For simplicity, we define an auxiliary variable $c$, which ranges between 0.01 and 0.1 , and $s=\lfloor c \times \min (M, N)\rfloor, w=2 s+1$, where $M$ and $N$ are the width and length of HSI data in the spatial domain. The influence of $c$ on final detection performance will be discussed in Section 3.2. Patch-image contains information on both background and anomalies, and they can be separated from each other via LRaSMD. The sparse matrix $\mathbf{S}$ corresponding to anomalies is exploited to induce the construction of the covariance matrix. It needs addressing that image reconstructed with matrix $\mathbf{S}$ can also be directly used as a detection map, which is not considered in the proposed FrHPI detector.

\section{Case Study}

In this section, we verify the efficiency and effectiveness of the proposed FrHPI detector for hyperspectral anomaly detection with four data sets. Two of the most widely used metrics for hyperspectral anomaly detection are used to evaluate the performance. The first one is the receiver 


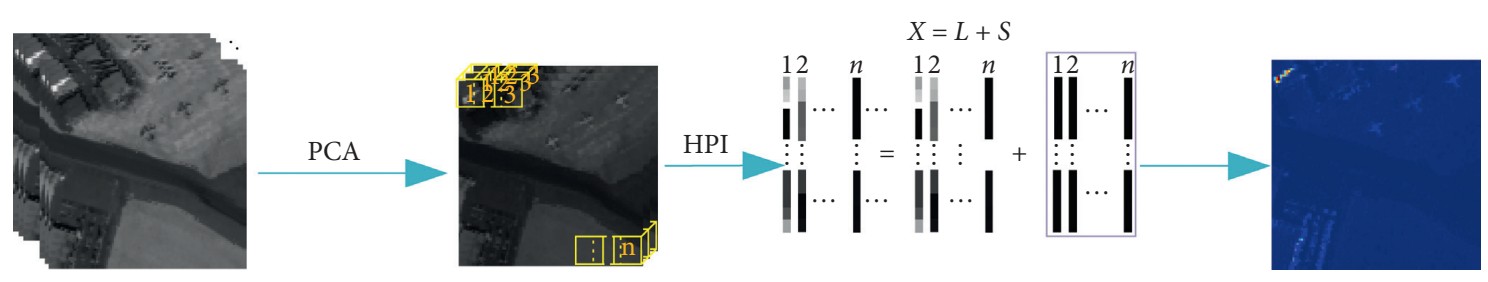

Figure 3: Hyperspectral patch-image model.

operating curve (ROC), which reveals the relationship between the detection probability $\left(P_{d}\right)$ and the false alarm rate $\left(P_{\mathrm{FAR}}\right)$. Specifically, $P_{d}$ and $P_{\mathrm{FAR}}$ are defined as follows:

$$
\begin{aligned}
P_{d} & =\frac{N_{\text {detected }}}{N_{\text {target }}}, \\
\mathrm{P}_{\text {FAR }} & =\frac{N_{\text {false }}}{N_{\text {background }}},
\end{aligned}
$$

where $N_{\text {detected }}$ and $N_{\text {target }}$ are the number of detected targets and the total target, while $N_{\text {false }}$ and $N_{\text {background }}$ are the numbers of false alarm pixels and the total background pixels in an image. If the ROC curve of a detector is at the top left of other detectors, this detector performs better than others. However, the ROC curve cannot quantitatively evaluate the detection performances. To this end, we introduce area under the curve (AUC) to overcome this problem. The AUC calculates the area under the ROC. Detectors with higher AUC values tend to detect targets with a lower false alarm rate. Moreover, the execution time is also calculated to evaluate the performance of detectors more comprehensively. All the experiments are implemented in MATLAB on an Intel Quad-Core i5-6200U CPU with 4 GB of RAM.

3.1. Data Sets. In order to verify the efficiency and effectiveness of the proposed FrHPI detector for hyperspectral anomaly detection, four data sets are tested. For simplicity, these four HSI data sets are renamed as HSI-1, HSI-2, HSI-3, and HSI-4. The details of these data sets are described as follows:

(1) Gulfport: it is derived from the airborne visible/infrared imaging spectrometer (AVIRIS) sensor, and this $100 \times 100$ HSI data set contains 191 spectral bands in wavelengths ranging from 400 to $2500 \mathrm{~nm}$ after removal of bad bands. This $3.5 \mathrm{~m}$ spatial resolution HSI contains three aircraft located at the bottom of the image, which is regarded as anomalies.

(2) EI Segundo: this data set was also captured by the AVIRIS sensor on the airborne platform. There are $250 \times 300$ pixels with a $7.1 \mathrm{~m}$ spatial resolution, while 224 spectral channels are preserved after bad bands are removed. The storage tanks and the towers are considered anomalies.

(3) San Diego-1: the $100 \times 100$ HSI data set was derived from the AVIRIS sensor. 189 bands have remained after the removal of water vapor absorption bands (1-6, 33-35, 97, 107-113, 156-66, and 221-224). It has a $20 \mathrm{~m}$ spatial resolution and $10 \mathrm{~nm}$ spectral resolution with wavelengths ranging from 370 to $2510 \mathrm{~nm}$. The three airplanes, which consist of 57 pixels, are treated as anomalies.

(4) San Diego-2: this image was captured from the AVIRIS sensor. The $200 \times 200 \times 189$ image has the same conditions as that of San Diego-1. There are three airplanes, which consist of 134 pixels, which are regarded as anomalies.

The pseudocolor images and the corresponding ground truth are illustrated in Figure 4.

3.2. Parameter Settings. The proposed FrHPI detector involves three parameters that need adjusting: the fractional transform order $p$, the window-related parameter $c$, and the positive tradeoff parameter $\lambda$. We analyze their influences on the final anomaly detection performance in this subsection. All four data sets are utilized here, and the AUC values are treated as the evaluation metric.

(1) Fractional transform order $p$ : the fractional transform order $p$ balances the information maintained from the original domain and its Fourier domain, and the transition with a great value of $p$ contains more Fourier information. Due to its ability to deal with nonstationary noise, FrFT can better distinguish anomalies from the background, which is beneficial to the detection result. Thus, it is essential to figure out the optimal values of $p$ to improve the detection performance. The window-related parameter $c$ is set to 0.05 , while the positive tradeoff parameter $\lambda$ is $10^{-2}$ for all four data sets. The range of the fractional transform order $p$ is set to $[0.1,0.2,0.3,0.4,0.5$, $0.6,0.7,0.8,0.9,1.0]$.

The AUC values with respect to $p$ are shown in Figure 5. It can be seen obviously that the parameter $p$ has a great influence on the detection performance in some data sets, especially in HSI-3 and HSI-4. Parameter $p$ determines the information preserved from the spectral domain and its Fourier domain. A proper choice of $p$ is beneficial to distinguishing anomalies from the background. Thus, the optimal 


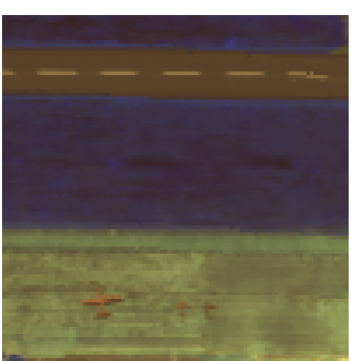

(a)

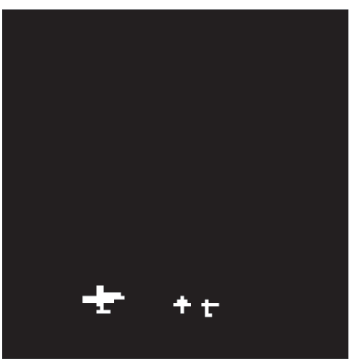

(e)

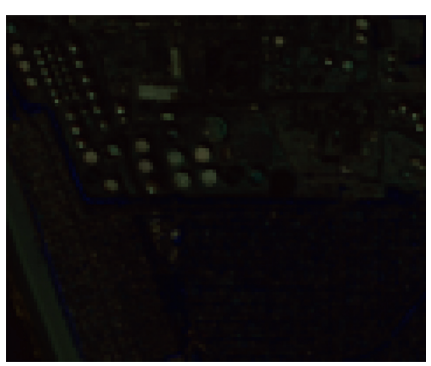

(b)

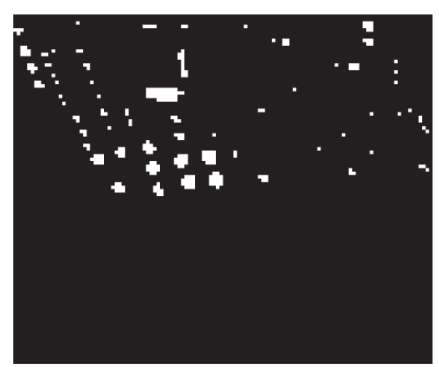

(f)

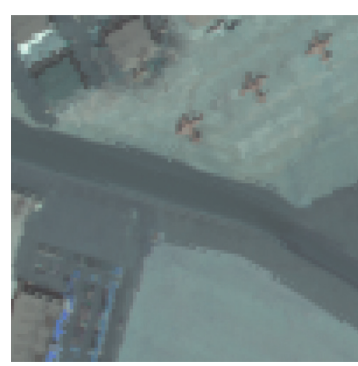

(c)

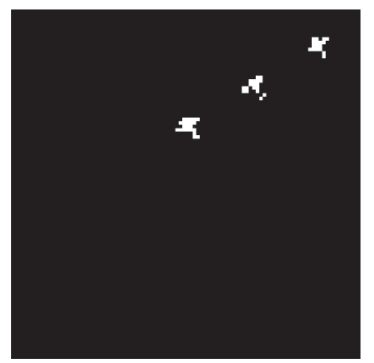

(g)

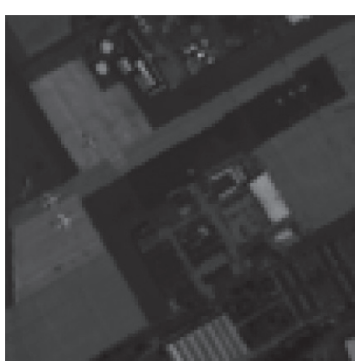

(d)

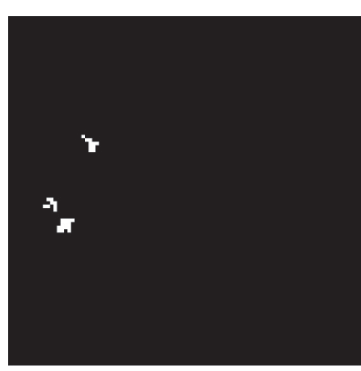

(h)

Figure 4: Pseudocolor images and the corresponding ground truth of the four data sets.

values of $p$ vary for all these data sets and are set to be $0.8,0.1,0.7$, and 0.9 , respectively.

(2) Window-related parameter $c$ : the window-related parameter $c$ controls the size $w$ and slide $s$ of the sliding rectangle window. These two parameters decide how much anomalous information is included in a patch-image $\mathbf{X}$ column, which significantly impacts the final detection performance. The positive tradeoff parameter $\lambda$ is set to $10^{-2}$ for all of the four data sets, and the fractional transform order $p$ is set to get the optimal results because the AUC values are sensitive to parameter $p$, as analyzed in (1). The range of the window-related parameter $c$ is set to $[0.01,0.02,0.03$, 0.04, 0.05, 0.06, 0.07, 0.08, 0.09, 0.1].

As demonstrated in Figure 6, the detection performance is sensitive to the window-related parameter $c$. The window-related parameter $c$ determines the size of patch-image $\mathbf{X}$, while parameter $c$ controls information preserved in each column of $\mathbf{X}$. The optimal $c$ encourages LRaSMD to separate the background from anomalies better. In this paper, $c$ for each of these four data sets are set to be 0.01, 0.04, 0.04 , and 0.10 .

(3) Tradeoff parameter $\lambda$ : the positive tradeoff parameter $\lambda$ in LRaSMD determines the rank function and the cardinality function's impact on the separation of background and anomalies. A proper setting of $\lambda$ provides satisfying masking for the construction of the background covariance matrix. Figure 7 demonstrates the separation of the lowrank part and sparse part when $\lambda$ is $10^{-4}, 10^{-2}$, and $10^{\circ}$, respectively. It is not hard to conclude that a

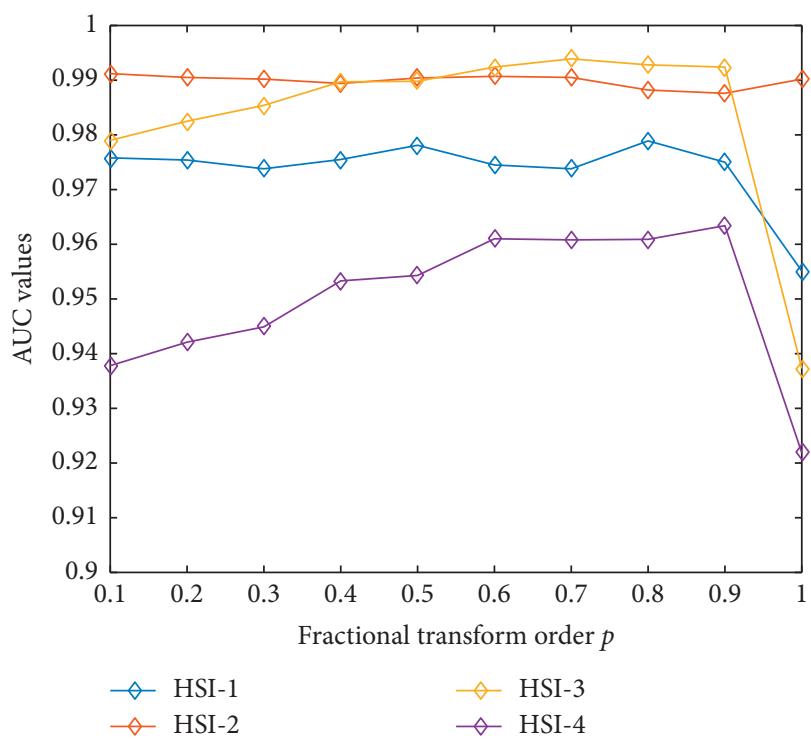

Figure 5: The impact of the fractional transform order $p$ on the final detection performance.

proper $\lambda$ encourages the background to cast aside the potential targets. To quantize the impact of $\lambda$ on the detection result, we set the fractional transform order $p$ and window-related parameter $c$ to get the optimal detection performance for all of the four data sets. The range of the positive tradeoff parameter $\lambda$ is set to $\left[10^{-5}, 10^{-4}, 10^{-3}, 10^{-2}, 10^{-1}, 10^{0}\right]$. The impact of the tradeoff parameter $\lambda$ on the final detection result is shown in Figure 8. The tradeoff parameter $\lambda$ balances information that the low-rank 


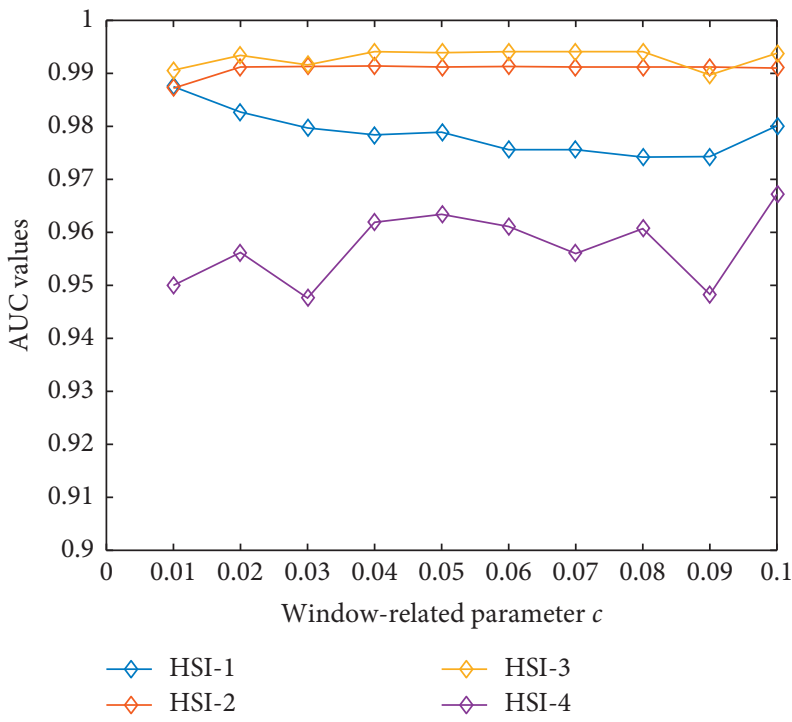

FIGURE 6: The impact of the window-related parameter $c$ on the final detection performance.

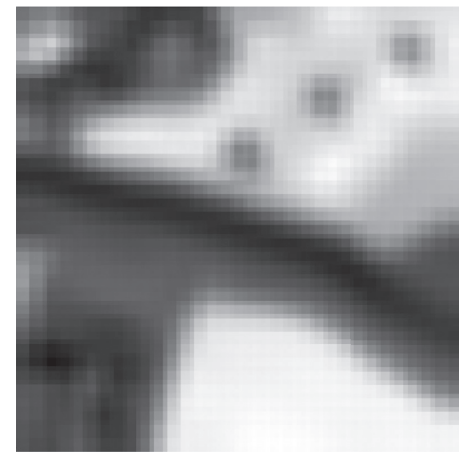

(a)

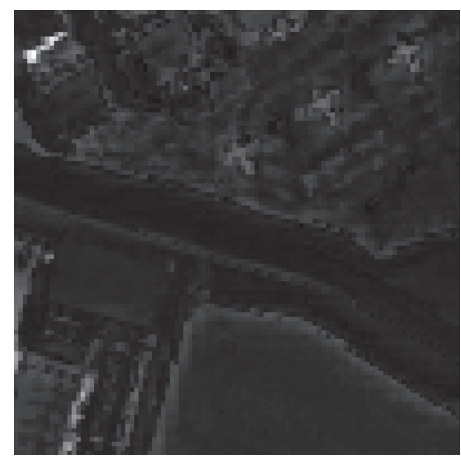

(d)

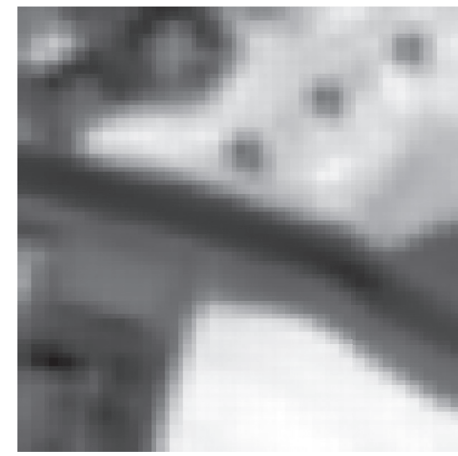

(b)

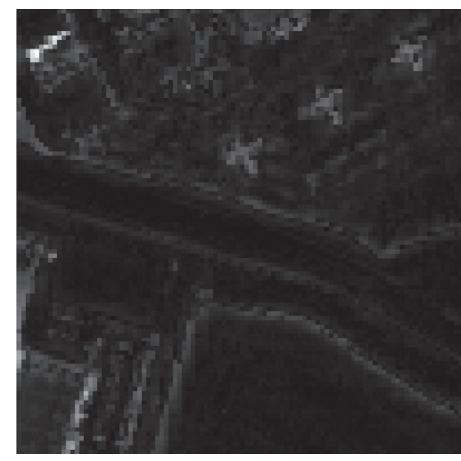

(e)

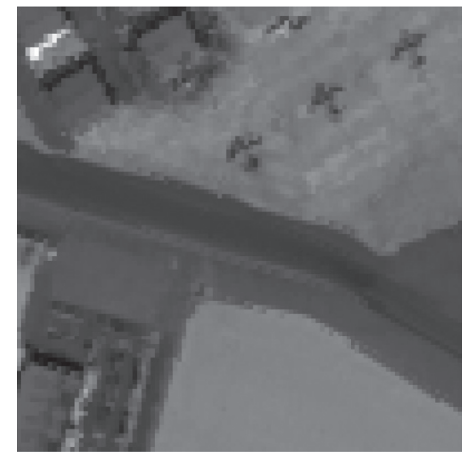

(c)

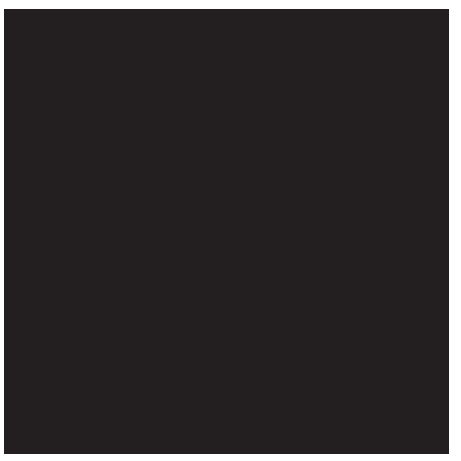

(f)

Figure 7: The low-rank part and sparse part when $\lambda$ is set to be $10^{-4}, 10^{-2}$, and $10^{0}$, respectively.

part and sparse part contain from the original data. The increase of $\lambda$ imposes a stronger penalty on the sparse part and vice versa. The optimal values of $\lambda$ for all of the data sets are set to be $10^{-2}$ for succinct purpose.

(4) Joint consideration of $p$ and $c$ : it is not hard to find that both the fractional transform order $p$ and window-related parameter $c$ vary in different data sets. Here, we jointly consider these two parameters to analyze their impacts on final detection performance further. The positive tradeoff parameter $\lambda$ is $10^{-2}$ for all of the four data sets. The range of $p$ and $c$ are set to $[0.1,0.2,0.3,0.4,0.5,0.6,0.7,0.8,0.9,1.0]$ and $[0.01,0.02,0.03,0.04,0.05,0.06,0.07,0.08,0.09$, $0.1]$, respectively.

Figure 9 reveals the detection performance when jointly considering $p$ and $c$. The proposed FrHPI detector is 


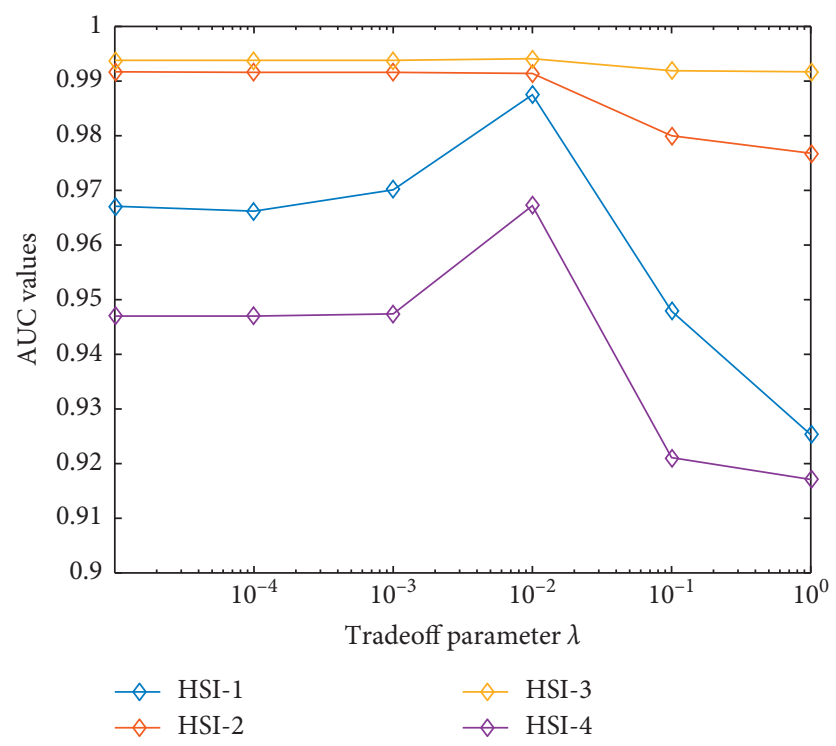

FIgURE 8: The impact of the tradeoff parameter $\lambda$ on the final detection performance.

relatively more sensitive to the fractional transform order $p$, and the lowest AUC value is obtained when $p=1$, which means that the spectrum is totally transformed into the Fourier domain. This verifies that traditional FT is not optimal when detecting targets in hyperspectral images, while a combination of signal domain and its Fourier domain can better separate the anomalies from the background. The window-related parameter $c$ also influences the performance, especially in data set HSI-4. Parameter $c$ controls the surrounding spatial information contains in each pixel under the test. Generally speaking, the proposed FrHPI detector performs well with most of these two parameters, as indicated by the flat areas with high tolerance.

3.3. Detection Performance. In this section, the hyperspectral anomaly detection performance of the proposed FrHPI detector is evaluated with six other traditional and state-ofthe-art detectors, which are described as follows:

(1) GRX [11]: global RX detector models the background with the inverse of the covariance matrix under the assumption that background obeys the multivariate normal distribution, and anomalies can be picked up via the Mahalanobis distance. GRX is now a benchmark in hyperspectral anomaly detection.

(2) LRX [12]: local RX detector is a derivation of GRX, which formulates a covariance matrix for each pixel under test with a dual-window. Samples inside the outer window while outside the inner window are selected out to construct the covariance matrix.

(3) CRD [16]: a collaborative-representation-based detector assumes that background can be linearly represented by its surrounding pixels, while anomalies cannot. The reconstruction error is utilized to measure the anomalous probability for the corresponding pixel.
(4) LSMAD [20]: LRaSMD-based Mahalanobis distance method separates anomalies from the background by exploring the low-rank prior knowledge of the background and LRaSMD technology. Then, the background covariance matrix is constructed, and anomalies can be detected by the Mahalanobis distance.

(5) FrFE [23]: fractional Fourier entropy-based hyperspectral anomaly detector obtains features in an intermediate domain between the original reflectance spectrum and its Fourier transform as FrFT can enhance the discrimination between anomalies and background. The fractional transform order $p$ is automatically obtained with Shannon entropy.

(6) HPI: in order to verify the statement that FrFT can better distinguish anomalies from the background, we remove FrFT from the proposed FrHPI model. The original HSI data are used to detect the anomalies.

The parameters for each detector are set to get the optimal detection performance. They are listed in Table 1.

To verify the efficiency and effectiveness of the proposed FrHPI detector for anomaly detection in HSI, three widely used metrics, ROC, AUC, and the execution time, are utilized here. The results are shown in Figure 10, Tables 2, and 3 . Hyperspectral anomaly detection maps on these four data sets are drawn in Figure 11.

The results show that the FrHPI can better suppress the background and highlight anomalies and yields higher AUC values than other detectors in most cases. Targets in hyperspectral images usually appear as several groups of pixels in the spatial domain. However, some anomaly detectors such as GRX, LSMAD, and FrFE neglect the importance of the spatial information, and their detection performances are limited. Due to the low spatial resolution 


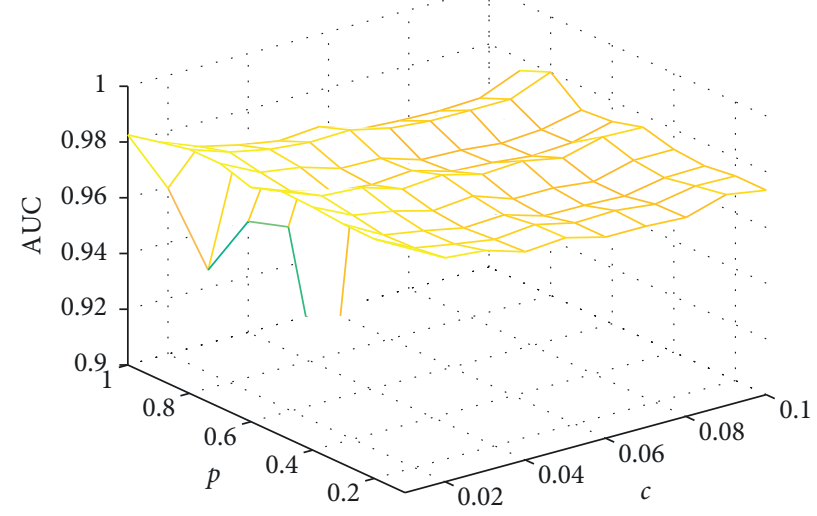

(a)

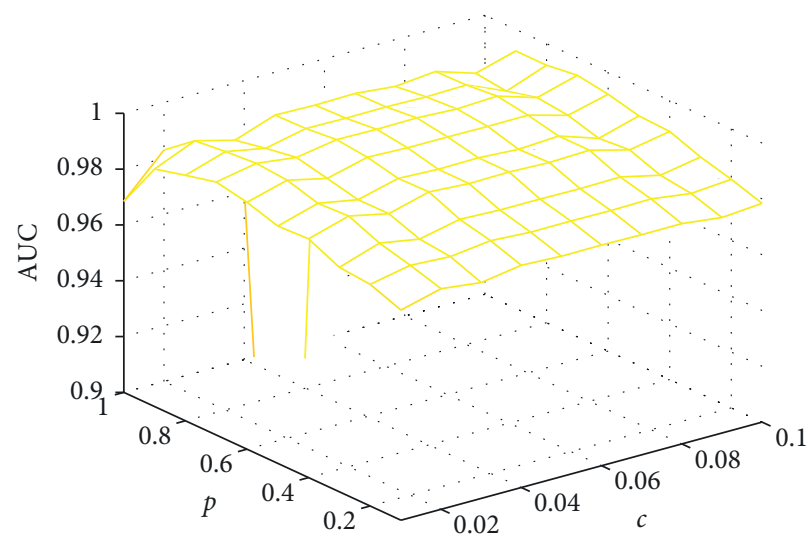

(c)

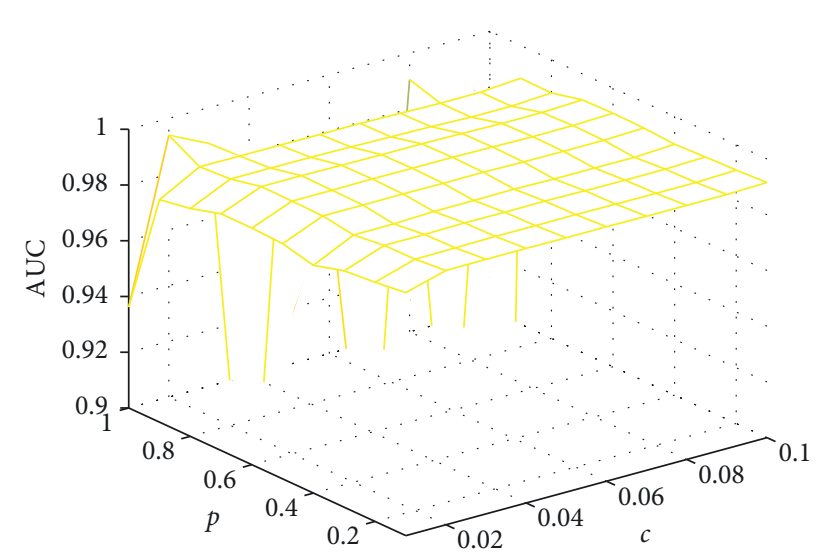

(b)

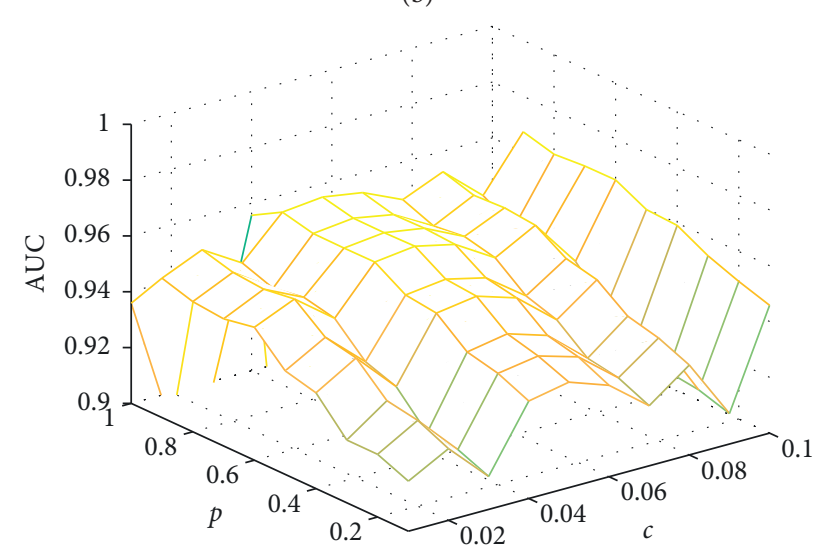

(d)

Figure 9: Joint consideration of $p$ and $c$ on the final detection performance: (a) HSI-1; (b) HSI-2; (c) HSI-3; (d) HSI-4.

TABLe 1: Parameter settings for these six hyperspectral anomaly detectors.

\begin{tabular}{|c|c|c|c|c|c|}
\hline Detector & Parameters & HSI-1 & HSI-2 & HSI-3 & HSI-4 \\
\hline GRX & \multicolumn{5}{|c|}{ There are no parameters need adjusting } \\
\hline LRX & $w_{\text {LRXin }} / w_{\text {LRXout }}$ & $17 / 19$ & $11 / 15$ & $13 / 17$ & $3 / 5$ \\
\hline CRD & $\begin{array}{c}\lambda_{\mathrm{CRD}} \\
w_{\mathrm{CRDin}} / w_{\mathrm{CRDout}}\end{array}$ & $\begin{array}{c}10^{-6} \\
21 / 23\end{array}$ & $\begin{array}{c}10^{-6} \\
19 / 21\end{array}$ & $\begin{array}{c}10^{-6} \\
13 / 15\end{array}$ & $\begin{array}{c}10^{-6} \\
19 / 21 \\
\end{array}$ \\
\hline LSMAD & $\begin{array}{l}r \\
k\end{array}$ & $\begin{array}{l}16 \\
0.7\end{array}$ & $\begin{array}{c}2 \\
0.1\end{array}$ & $\begin{array}{c}2 \\
0.9\end{array}$ & $\begin{array}{c}2 \\
0.6\end{array}$ \\
\hline FrFE & $p_{\text {FrFE }}$ & 0.8 & 0.1 & 0.7 & 0.9 \\
\hline HPI & $\begin{array}{l}c_{\mathrm{HPI}} \\
\lambda_{\mathrm{HPI}}\end{array}$ & $\begin{array}{l}0.02 \\
10^{-2} \\
\end{array}$ & $\begin{array}{l}0.06 \\
10^{-2} \\
\end{array}$ & $\begin{array}{l}0.06 \\
10^{-2} \\
\end{array}$ & $\begin{array}{l}0.10 \\
10^{-2} \\
\end{array}$ \\
\hline FrHPI & $\begin{array}{l}c_{\text {FrHPI }} \\
\lambda_{\text {FrHPI }} \\
p_{\text {FrHPI }}\end{array}$ & $\begin{array}{c}0.01 \\
10^{-2} \\
0.8\end{array}$ & $\begin{array}{c}0.04 \\
10^{-2} \\
0.1 \\
\end{array}$ & $\begin{array}{c}0.04 \\
10^{-2} \\
0.7 \\
\end{array}$ & $\begin{array}{c}0.10 \\
10^{-2} \\
0.9 \\
\end{array}$ \\
\hline
\end{tabular}

and corruption of noise, the background may share similar patterns with anomalies. Although LRX and CRD consider the spatial information, they detect anomalies with the raw data without feature extraction. The ability of FrFT feature extraction in our method is verified, as the results show that
FrHPI detects all the anomalous pixels under a lower false alarm rate than HPI. A comparison with six hyperspectral anomaly detectors confirms the superiority of the proposed FrHPI detector. Moreover, the execution time of FrHPI is also acceptable as shown in Table 3. 


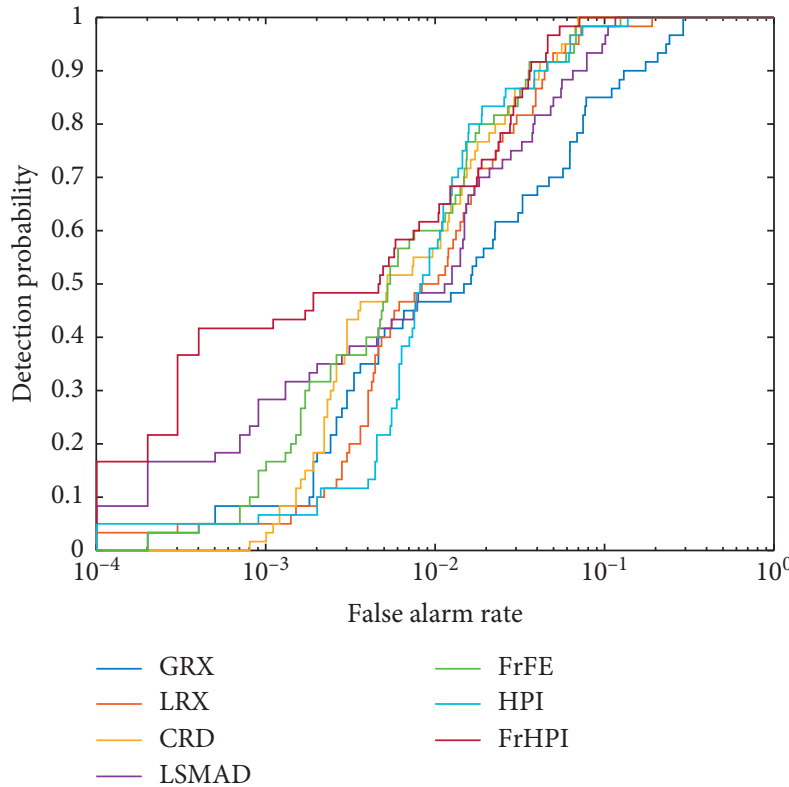

(a)

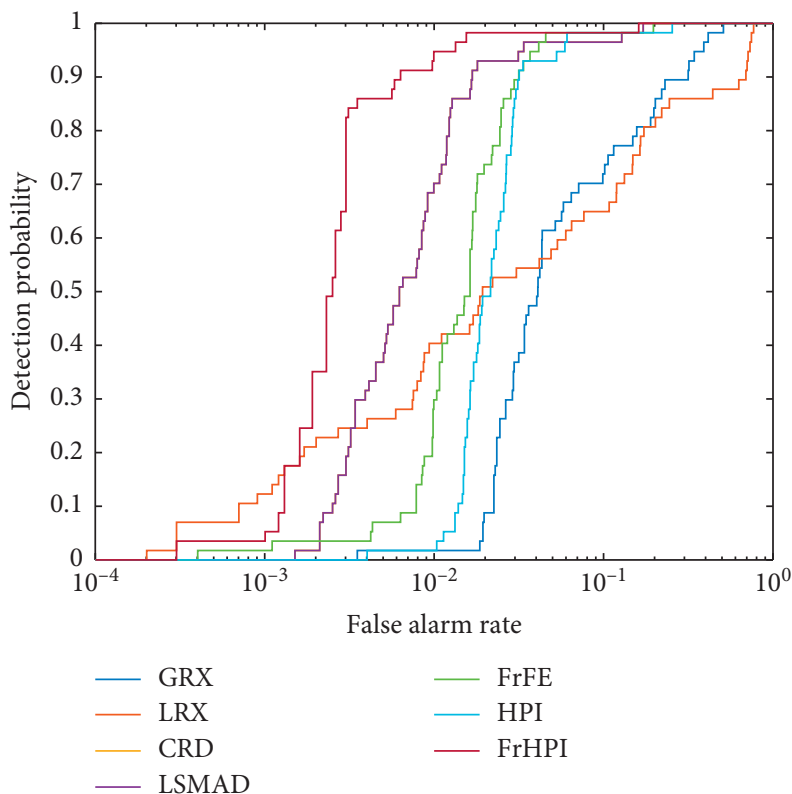

(c)

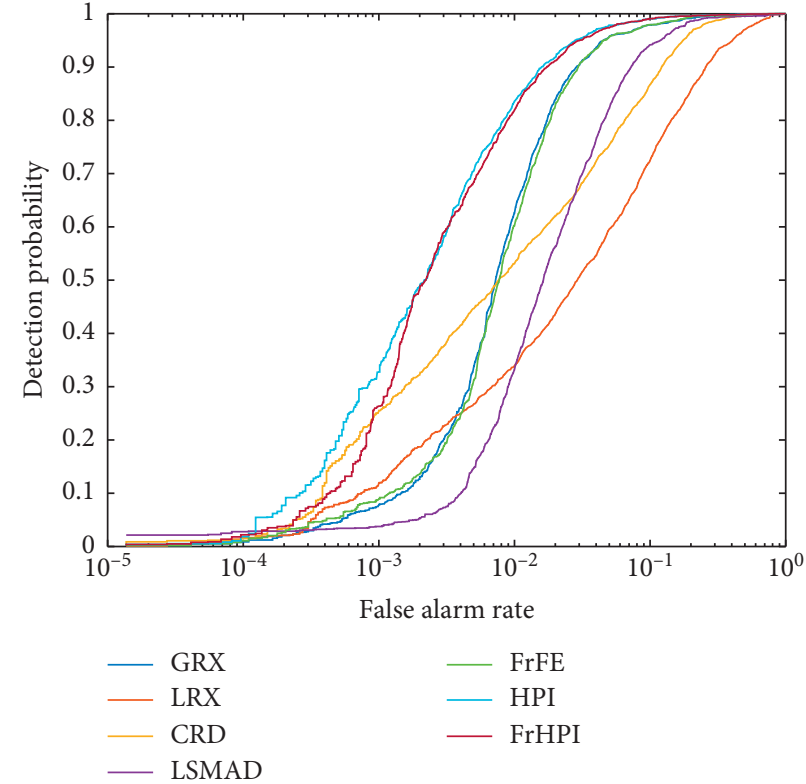

(b)

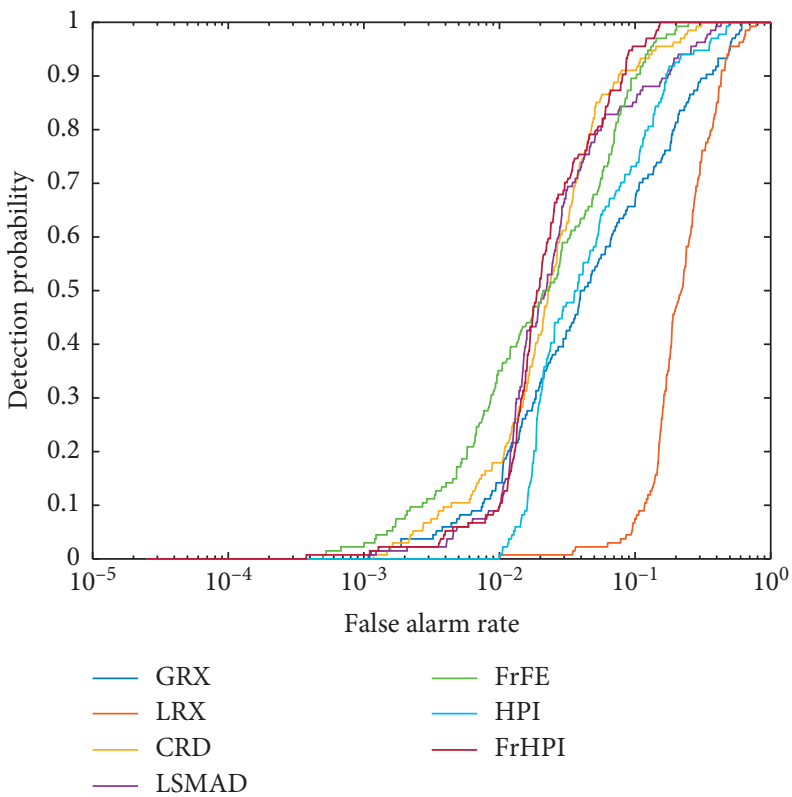

(d)

FIgURE 10: The ROC curves on four hyperspectral data sets.

TABLE 2: The AUC values on four data sets.

\begin{tabular}{lllll}
\hline Detector & HSI-1 & HSI-2 & HSI-3 & \\
\hline GRX & 0.9526 & 0.9841 & 0.9055 & 0.8870 \\
LRX & 0.9810 & 0.9099 & 0.8605 & 0.7464 \\
CRD & 0.9858 & 0.9603 & 0.9870 & 0.9607 \\
LSMAD & 0.9780 & 0.9659 & 0.9805 & 0.9464 \\
FrFE & 0.9853 & 0.9839 & 0.9805 & 0.9591 \\
HPI & 0.9838 & 0.9916 & 0.9737 & 0.9221 \\
FrHPI & 0.9875 & 0.9914 & 0.9941 & 0.9672 \\
\hline
\end{tabular}


TABLE 3: The execution time (in second) for four data sets.

\begin{tabular}{lcccc}
\hline Detector & HSI-1 & HSI-2 & HSI-3 & \\
GRX & 0.3 & 1.8 & 0.2 & \\
LRX & 63.8 & 662.6 & 74.4 & 19.7 \\
CRD & 39.8 & 226.2 & 16.7 & 109.5 \\
LSMAD & 65.3 & 418.2 & 65.0 & 2.3 \\
FrFE & 1.4 & 10.2 & 1.2 & 4.9 \\
HPI & 2.1 & 13.7 & 2.7 & 4.5 \\
FrHPI & 5.4 & 50.0 & 5.1 & 9.3 \\
\hline
\end{tabular}
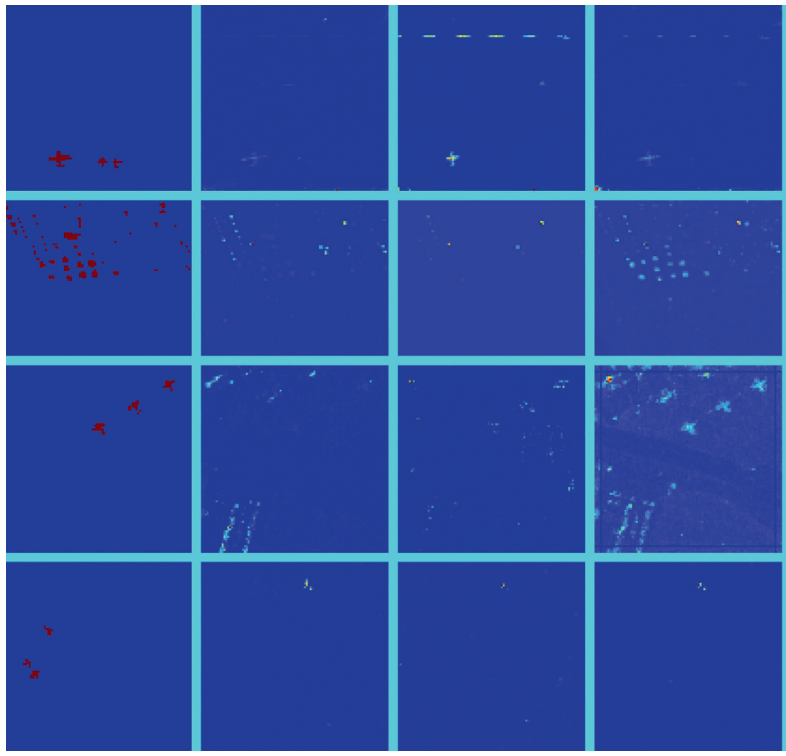
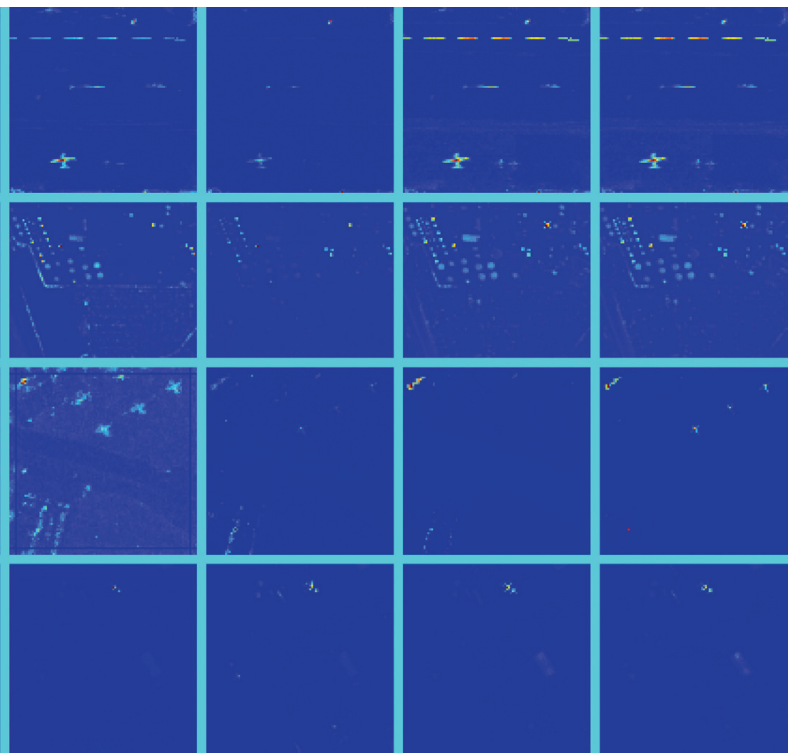

FIGURE 11: Hyperspectral anomaly detection maps on four data sets. From top to bottom: HSI-1, HSI-2, HSI-3, and HSI-4. From left to right: ground truth, GRX, LRX, CRD, LSMAD, FrFE, HPI, and FrHPI.

\section{Conclusions}

In this paper, a FrHPI detector is proposed for anomaly detection in hyperspectral images. The HPI model is proposed to separate the anomalies, and LRaSMD is employed to induce the formulation of the covariance matrix. Meanwhile, FrFT is utilized as a preprocessing step to distinguish anomalies from the background better. In the end, the detection map is obtained with Mahalanobis distance.

Extensive experiments are conducted to demonstrate the effectiveness and efficiency of the FrHPI detector in four hyperspectral data sets. The average AUC improvement is $3.8 \%$ on these four data sets, and the time cost is also acceptable for general anomaly detection tasks. Although the proposed detector has satisfying detection performance compared with other anomaly detectors, it is still challenging to automatically adjust the optimal parameters, which will be studied in our future work.

\section{Data Availability}

The data used to support the findings of this study are available from the corresponding author upon request.

\section{Conflicts of Interest}

The authors declare that there are no conflicts of interest regarding the publication of this paper.

\section{Acknowledgments}

This research was funded by the National Natural Science Foundation of China (NSFC, Grant No. 61705170), the NSFC-CAAC Joint Fund (Grant No. U1833119), Natural Science Foundation of Hubei Province (Grant No. 2020CFA063), Wuhan Science and Technology Foundation (Grant No. 2018020401011299), and National Food and Strategic Reserves Administration Foundation (Grant No. LQ2018501).

\section{References}

[1] J. M. Bioucas-Dias, A. Plaza, G. Camps-Valls, P. Scheunders, N. Nasrabadi, and J. Chanussot, "Hyperspectral remote sensing data analysis and future challenges," IEEE Geoscience and Remote Sensing Magazine, vol. 1, no. 2, pp. 6-36, 2013.

[2] H. Li, C. Li, C. Zhang, Z. Liu, and C. Liu, "Hyperspectral image classification with spatial filtering and $l_{2,1}$ norm," Sensors, vol. 17, no. 2, p. 314, 2017. 
[3] X. Mei, Y. Ma, C. Li, F. Fan, J. Huang, and J. Ma, "Robust GBM hyperspectral image unmixing with superpixel segmentation based low rank and sparse representation," Neurocomputing, vol. 275, pp. 2783-2797, 2018.

[4] L. Zhang, L. Zhang, D. Tao et al., "Sparse transfer manifold embedding for hyperspectral target detection," IEEE Transactions on Geoscience and Remote Sensing, vol. 52, no. 2, pp. 1030-1043, 2013.

[5] J. Yue, G. Yang, C. Li et al., "Estimation of winter wheat above-ground biomass using unmanned aerial vehicle-based snapshot hyperspectral sensor and crop height improved models," Remote Sensing, vol. 9, no. 7, p. 708, 2017.

[6] P. Arellano, K. Tansey, H. Balzter, and D. S. Boyd, "Detecting the effects of hydrocarbon pollution in the amazon forest using hyperspectral satellite images," Environmental Pollution, vol. 205, pp. 225-239, 2015.

[7] N. Li, X. Huang, H. Zhao et al., "Multiparameter optimization for mineral mapping using hyperspectral imagery," IEEE Journal of Selected Topics in Applied Earth Observations and Remote Sensing, vol. 11, no. 4, pp. 1348-1357, 2018.

[8] T. A. Carrino, A. P. Crósta, C. L. B. Toledo, and A. M. Silva, "Hyperspectral remote sensing applied to mineral exploration in Southern Peru: a multiple data integration approach in the Chapi Chiara gold prospect," International Journal of Applied Earth Observation and Geoinformation, vol. 64, pp. 287-300, 2018.

[9] M. Shimoni, R. Haelterman, and C. Perneel, "Hypersectral imaging for military and security applications: combining myriad processing and sensing techniques," IEEE Geoscience and Remote Sensing Magazine, vol. 7, no. 2, pp. 101-117, 2019.

[10] Y. Ma, G. Fan, Q. Jin et al., "Hyperspectral anomaly detection via integration of feature extraction and background purification," IEEE Geoscience and Remote Sensing Letters, pp. 1-5, 2020.

[11] Y. Ma, Q. Jin, X. Mei et al., "Hyperspectral unmixing with Gaussian mixture model and low-rank representation," Remote Sensing, vol. 11, no. 8, 2019.

[12] Y. Qu, W. Wang, R. Guo et al., "Hyperspectral anomaly detection through spectral unmixing and dictionary-based low-rank decomposition," IEEE Transactions on Geoscience and Remote Sensing, vol. 56, no. 8, pp. 4391-4405, 2018.

[13] N. Huyan, X. Zhang, H. Zhou, and L. Jiao, "Hyperspectral anomaly detection via background and potential anomaly dictionaries construction," IEEE Transactions on Geoscience and Remote Sensing, vol. 57, no. 4, pp. 2263-2276, 2019.

[14] I. S. Reed and X. Yu, "Adaptive multiple-band CFAR detection of an optical pattern with unknown spectral distribution," IEEE Transactions on Acoustics, Speech, and Signal Processing, vol. 38, no. 10, pp. 1760-1770, 1990.

[15] J. M. Molero, E. M. Garzon, I. Garcia, and A. Plaza, “Analysis and optimizations of global and local versions of the RX algorithm for anomaly detection in hyperspectral data," IEEE Journal of Selected Topics in Applied Earth Observations and Remote Sensing, vol. 6, no. 2, pp. 801-814, 2013.

[16] H. Kwon and N. M. Nasrabadi, "Kernel RX-algorithm: a nonlinear anomaly detector for hyperspectral imagery," IEEE Transactions on Geoscience and Remote Sensing, vol. 43, no. 2, pp. 388-397, 2005.

[17] S. Chang, B. Du, and L. Zhang, "A sparse autoencoder based hyperspectral anomaly detection algorihtm using residual of reconstruction error," in Proceedings of the IEEE International Geoscience and Remote Sensing Symposium (IGARSS), pp. 5488-5491, Seoul, Korea, July 2019.

[18] W. Li and Q. Du, "Collaborative representation for hyperspectral anomaly detection," IEEE Transactions on Geoscience and Remote Sensing, vol. 53, no. 3, pp. 1463-1474, 2015.
[19] S. Song, H. Zhou, Y. Yang, and J. Song, "Hyperspectral anomaly detection via convolutional neural network and low rank with density-based clustering," IEEE Journal of Selected Topics in Applied Earth Observations and Remote Sensing, vol. 12, no. 9, pp. 3637-3649, 2019.

[20] C. Zhao and L. Zhang, "Spectral-spatial stacked autoencoders based on low-rank and sparse matrix decomposition for hyperspectral anomaly detection," Infrared Physics \& Technology, vol. 92, pp. 166-176, 2018.

[21] Y. Xu, Z. Wu, J. Li et al., "Anomaly detection in hyperspectral images based on low-rank and sparse representation," IEEE Transactions on Geoscience and Remote Sensing, vol. 54, no. 4, pp. 1990-2000, 2016.

[22] Y. Zhang, B. Du, L. Zhang, and S. Wang, "A low-rank and sparse matrix decomposition-based Mahalanobis distance method for hyperspectral anomaly detection," IEEE Transactions on Geoscience and Remote Sensing, vol. 54, no. 3, pp. 1376-1389, 2016.

[23] Y. Xu, B. Du, L. Zhang, and S. Chang, "A low-rank and sparse matrix decomposition- based dictionary reconstruction and anomaly extraction framework for hyperspectral anomaly detection," IEEE Geoscience and Remote Sensing Letters, vol. 17, no. 7, pp. 1248-1252, 2020.

[24] Y. Peng, Z. Wang, K. Jiang, Z. Shao, and J. Ma, "Multi-temporal ultra dense memory network for video super-resolution," IEEE Transactions on Circuits and Systems for Video Technology, vol. 30, no. 8, pp. 2503-2516, 2020.

[25] X. Lu, W. Zhang, and J. Huang, "Exploiting embedding manifold of autoencoders for hyperspectral anomaly detection," IEEE Transactions on Geoscience and Remote Sensing, vol. 58, no. 3, pp. 1527-1537, 2020.

[26] C. Gao, D. Meng, Y. Yang, Y. Wang, X. Zhou, and A. G. Hauptmann, "Infrared patch-image model for small target detection in a single image," IEEE Transactions on Image Processing, vol. 22, no. 12, pp. 4996-5009, 2013.

[27] R. Tao, X. Zhao, W. Li, H.-C. Li, and Q. Du, "Hyperspectral anomaly detection by fractional Fourier entropy," IEEE Journal of Selected Topics in Applied Earth Observations and Remote Sensing, vol. 12, no. 12, pp. 4920-4929, 2019.

[28] J.-F. Cai, E. J. Candès, and Z. Shen, "A singular value thresholding algorithm for matrix completion," SIAM Journal on Optimization, vol. 20, no. 4, pp. 1956-1982, 2010.

[29] D. L. Donoho, "De-noising by soft-thresholding," IEEE Transactions on Information Theory, vol. 41, no. 3, pp. 613-627, 1995.

[30] A. Rohman and Y. B. C. Man, "Fourier transform infrared (FTIR) spectroscopy for analysis of extra virgin olive oil adulterated with palm oil," Food Research International, vol. 43, no. 3, pp. 886-892, 2010.

[31] C. Candan, M. A. Kutay, and H. M. Ozaktas, "The discrete fractional Fourier transform," IEEE Transactions on Signal Processing, vol. 48, no. 5, pp. 1329-1337, 2000.

[32] S. Chen, S. Zhang, H. Zhao, and Y. Chen, "A new chirp scaling algorithm for highly squinted missile-borne SAR based on FrFT," IEEE Journal of Selected Topics in Applied Earth Observations and Remote Sensing, vol. 8, no. 8, pp. 3977-3987, 2015.

[33] J. B. Campbell, F. Perez, Q. Wang et al., "Remote vibration estimation using displaced-phase-center antenna SAR for strong clutter environments," IEEE Transactions on Geoscience and Remote Sensing, vol. 56, no. 5, pp. 2735-2747, 2018.

[34] Q. Wang, Q. Guo, J. Zhou, and Q. Lin, "Nonlinear joint fractional Fourier transform correlation for target detection in hyperspectral image," Optics \& Laser Technology, vol. 44, no. 6, pp. 1897-1904, 2012. 